The Neurobiology of Opioid Dependence: Implications for Treatment

Thomas R. Kosten, M.D.1,2

Tony P. George, M.D. ${ }^{1,3}$

'Yale University School of Medicine New Haven, Connecticut

2 VA Connecticut Healthcare System

West Haven, Connecticut

3 Connecticut Mental Health Center New Haven, Connecticut
Opioid tolerance, dependence, and addiction are all manifestations of brain changes resulting from chronic opioid abuse. The opioid abuser's struggle for recovery is in great part a struggle to overcome the effects of these changes. Medications such as methadone, LAAM, buprenorphine, and naltrexone act on the same brain structures and processes as addictive opioids, but with protective or normalizing effects. Despite the effectiveness of medications, they must be used in conjunction with appropriate psychosocial treatments.
W hile the individual patient, rather than his or her disease, is the appropriate focus of treatment for opioid abuse, an understanding of the neurobiology of dependence and addiction can be invaluable to the clinician. It can provide insight about patient behaviors and problems, help define realistic expectations, and clarify the rationales for treatment methods and goals. As well, patients who are informed about the brain origins of addiction can benefit from understanding that their illness has a biological basis and does not mean they are "bad" people.

Brain abnormalities resulting from chronic use of heroin, oxycodone, and other morphine-derived drugs are underlying causes of opioid dependence (the need to keep taking drugs to avoid a withdrawal syndrome) and addiction (intense drug craving and compulsive use). The abnormalities that produce dependence, well understood by science, appear to resolve after detoxification, within days or weeks after opioid use stops. The abnormalities that produce addiction, however, are more wide-ranging, complex, and long-lasting. They may involve an interaction of environmental effects-for example, stress, the social context of initial opiate use, and psychological conditioning — and a genetic predisposition in the form of brain pathways that were abnormal even before the first dose of opioid was taken. Such abnormalities can produce craving that leads to relapse months or years after the individual is no longer opioid dependent.

In this article we describe how opioids affect brain processes to produce drug liking, tolerance, dependence, and addiction. While these processes, like everything else that happens in the brain, are highly complex, we try to explain them in terms that can be easily understood and explained to patients. We also discuss 
FIGURE 1. The Mesolimbic Reward System

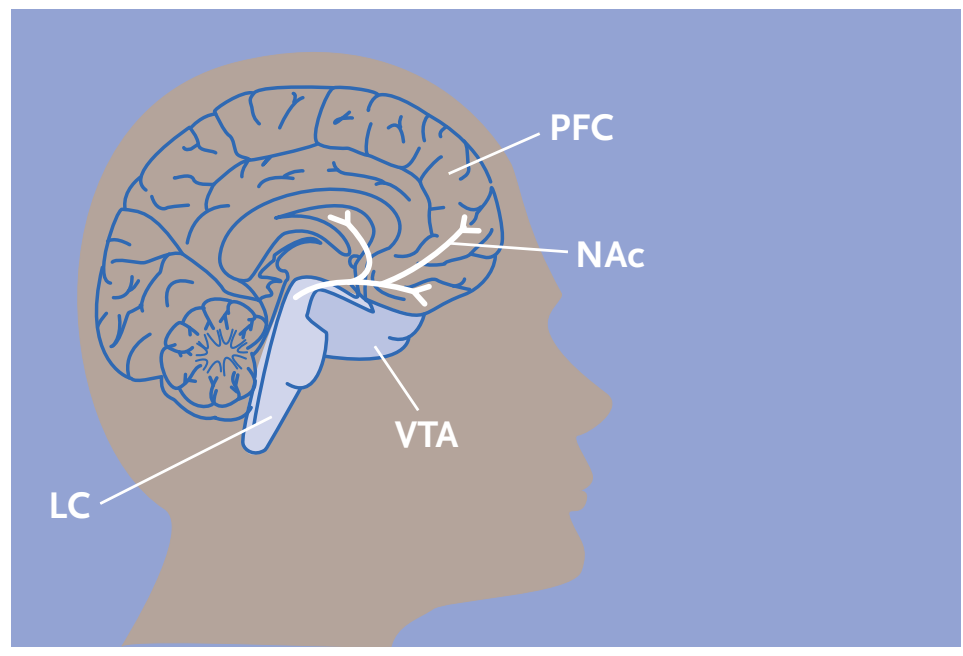

When drugs stimulate mu opioid receptors in the brain, cells in the ventral tegmental area (VTA) produce dopamine and release it into the nucleus accumbens (NAc), giving rise to feelings of pleasure. Feedback from the prefrontal cortex (PFC) to the VTA helps us overcome drives to obtain pleasure through actions that may be unsafe or unwise, but this feedback appears to be compromised in individuals who become addicted to drugs. The locus ceruleus (LC) is an area of the brain that plays an important role in drug dependence.

benefit from understanding that their addiction has a biological basis. the treatment implications of these concepts. Pharmacological therapy with methadone, LAAM (levo-alpha-acetylmethadol), naltrexone, or other medications directly offsets or reverses some of the brain changes associated with addiction, greatly enhancing the effectiveness of behavioral therapies. Although researchers do not yet know everything about how these medications work, it is clear that they are all truly active treatments, rather than simply substitutes for the addictive opioids.

\section{ORIGINS OF DRUG LIKING}

Many factors, both individual and environmental, influence whether a particular person who experiments with opioid drugs will continue taking them long enough to become dependent or addicted. For individuals who do continue, the opioids' ability to provide intense feelings of pleasure is a critical reason.

When heroin, oxycodone, or any other opiate travels through the bloodstream to the brain, the chemicals attach to specialized proteins, called mu opioid receptors, on the surfaces of opiate-sensitive neu- rons (brain cells). The linkage of these chemicals with the receptors triggers the same biochemical brain processes that reward people with feelings of pleasure when they engage in activities that promote basic life functions, such as eating and sex. Opioids are prescribed therapeutically to relieve pain, but when opioids activate these reward processes in the absence of significant pain, they can motivate repeated use of the drug simply for pleasure.

One of the brain circuits that is activated by opioids is the mesolimbic (midbrain) reward system. This system generates signals in a part of the brain called the ventral tegmental area (VTA) that result in the release of the chemical dopamine (DA) in another part of the brain, the nucleus accumbens (NAc) (Figure 1). This release of DA into the NAc causes feelings of pleasure. Other areas of the brain create a lasting record or memory that associates these good feelings with the circumstances and environment in which they occur. These memories, called conditioned associations, often lead to the craving for drugs when the abuser reencounters those persons, places, or things, and they drive abusers to seek out more drugs in spite of many obstacles.

Particularly in the early stages of abuse, the opioid's stimulation of the brain's reward system is a primary reason that some people take drugs repeatedly. However, the compulsion to use opioids builds over time to extend beyond a simple drive for pleasure. This increased compulsion is related to tolerance and dependence.

\section{OPIOID TOLERANCE, DEPENDENCE, AND WITHDRAWAL}

From a clinical standpoint, opioid withdrawal is one of the most powerful factors driving opioid dependence and addictive behaviors. Treatment of the patient's withdrawal symptoms is based on understanding how withdrawal is related to the brain's adjustment to opioids.

Repeated exposure to escalating dosages of opioids alters the brain so that it functions more or less normally when the drugs are present and abnormally when they are not. Two clinically important results of this alteration are opioid tolerance (the need to take higher and higher dosages of drugs to achieve the same opioid effect) and drug dependence (susceptibility to withdrawal symptoms). Withdrawal symptoms occur only in patients who have developed tolerance. 
Opioid tolerance occurs because the brain cells that have opioid receptors on them gradually become less responsive to the opioid stimulation. For example, more opioid is needed to stimulate the VTA brain cells of the mesolimbic reward system to release the same amount of DA in the NAc. Therefore, more opioid is needed to produce pleasure comparable to that provided in previous drug-taking episodes.

Opioid dependence and some of the most distressing opioid withdrawal symptoms stem from changes in another important brain system, involving an area at the base of the brain - the locus ceruleus (LC) (Figure 2). Neurons in the LC produce a chemical, noradrenaline (NA), and distribute it to other parts of the brain where it stimulates wakefulness, breathing, blood pressure, and general alertness, among other functions. When opioid molecules link to mu receptors on brain cells in the LC, they suppress the neurons' release of NA, resulting in drowsiness, slowed respiration, low blood pressure-familiar effects of opioid intoxication. With repeated exposure to opioids, however, the LC neurons adjust by increasing their level of activity. Now, when opioids are present, their suppressive impact is offset by this heightened activity, with the result that roughly normal amounts of NA are released and the patient feels more or less normal. When opioids are not present to suppress the LC brain cells' enhanced activity, however, the neurons release excessive amounts of NA, triggering jitters, anxiety, muscle cramps, and diarrhea.

Other brain areas in addition to the $\mathrm{LC}$ also contribute to the production of withdrawal symptoms, including the mesolimbic reward system. For example, opioid tolerance that reduces the VTA's release of DA into the NAc may prevent the patient from obtaining pleasure from normally rewarding activities such as eating. These changes in the VTA and the DA reward systems, though not fully understood, form an important brain system underlying craving and compulsive drug use.

\section{TRANSITION TO ADDICTION}

As we have seen, the pleasure derived from opioids' activation of the brain's natural reward system promotes continued drug use during the initial stages of opioid addiction. Subsequently, repeated exposure to opioid drugs induces the brain mechanisms of dependence, which leads to daily drug use to avert the unpleasant symptoms of drug withdrawal. Further

\section{Definitions of Key Terms}

dopamine (DA): A neurotransmitter present in brain regions that regulate movement, emotion, motivation, and the feeling of pleasure.

GABA (gamma-amino butyric acid): A neurotransmitter in the brain whose primary function is to inhibit the firing of neurons.

locus ceruleus (LC): A region of the brain that receives and processes sensory signals from all areas of the body; involved in arousal and vigilance.

noradrenaline (NA): A neurotransmitter produced in the brain and peripheral nervous system; involved in arousal and regulation of blood pressure, sleep, and mood; also called norepinephrine.

nucleus accumbens (NAc): A structure in the forebrain that plays an important part in dopamine release and stimulant action; one of the brain's key pleasure centers.

prefrontal cortex (PFC): The frontmost part of the brain; involved in higher cognitive functions, including foresight and planning.

ventral tegmental area (VTA): The group of dopamine-containing neurons that make up a key part of the brain reward system; key targets of these neurons include the nucleus accumbens and the prefrontal cortex

prolonged use produces more long-lasting changes in the brain that may underlie the compulsive drug-seeking behavior and related adverse consequences that are the hallmarks of addiction. Recent scientific research has generated several models to explain how habitual drug use produces changes in the brain that may lead to drug addiction. In reality, the process of addiction probably involves components from each of these models, as well as other features.

\section{The "Changed Set Point" Model}

The "changed set point" model of drug addiction has several variants based on the altered neurobiology of the DA neurons in the VTA and of the NA neurons of the LC during the early phases of withdrawal and abstinence. The basic idea is that drug abuse alters a biological or physiological setting or baseline. One variant, by Koob and LeMoal (2001), is based on the idea that neurons of the mesolimbic reward pathways are naturally "set" to release enough DA in the NAc to produce a normal level of pleasure. Koob and LeMoal suggest that opioids cause addiction by initiating a 
Opioid toler-

ance occurs

because the

brain cells

gradually

become less

responsive to

the opioid stim.

ulation. vicious cycle of changing this set point such that the release of DA is reduced when normally pleasurable activities occur and opioids are not present. Similarly, a change in set point occurs in the LC, but in the opposite direction, such that NA release is increased during withdrawal, as described above. Under this model, both the positive (drug liking) and negative (drug withdrawal) aspects of drug addiction are accounted for.

A specific way that the DA neurons can become dysfunctional relates to an alteration in their baseline ("resting") levels of electrical activity and DA release (Grace, 2000). In this second variant of the changed set point model, this resting level is the result of two factors that influence the amount of resting DA release in the NAc: cortical excitatory (glutamate) neurons that drive the VTA DA neurons to release DA, and autoreceptors ("brakes") that shut down further release when DA concentrations become excessive. Activation of opioid receptors by heroin and heroin-like drugs initially bypasses these brakes and leads to a large release of DA in the NAc. However, with repeated heroin use, the brain responds to these successive large DA releases by increasing the number and strength of the brakes on the VTA DA neurons. Eventually, these enhanced "braking" autoreceptors inhibit the neurons' resting DA release. When this happens, the dependent addict will take even more heroin to offset the reduction of normal resting DA release. When he or she stops the heroin use, a state of DA deprivation will result, manifesting in dysphoria (pain, agitation, malaise) and other withdrawal symptoms, which can lead to a cycle of relapse to drug use.

A third variation on the set-point change emphasizes the sensitivity to environmental cues that leads to drug wanting or craving rather than just reinforcement and withdrawal (Breiter et al., 1997; Robinson and Berridge, 2000). During periods when the drug is not available to addicts, their brains can remember the drug, and desire or craving for the drug can be a major factor leading to drug use relapse. This craving may represent increased activity of the cortical excitatory (glutamate) neurotransmitters, which drive the resting activity of the DA-containing VTA neurons, as mentioned, and also drive the LC NA neurons. As the glutamate activity increases, DA will be released from the VTA, leading to drug wanting or craving, and NA will be released from the LC, leading to increased opioid withdrawal symptoms. This theory suggests that these cortical excitatory brain pathways are overactive in heroin addiction and that reducing their activity would be therapeutic. Scientists are currently researching a medication called lamotrigene and related compounds called excitatory amino acid antagonists to see whether this potential treatment strategy really can work.

Thus, several mechanisms in the LC and VTANAc brain pathways may be operating during addiction and relapse. The excitatory cortical pathways may produce little response in the VTA during the resting state, leading to reductions in DA. However, when the addicted individual is exposed to cues that produce craving, the glutamate pathways may get sufficiently active to raise DA and stimulate desire for a greater high. This same increase in glutamate activity will raise NA release from the LC to produce a dysphoric state predisposing to relapse and continued addiction.

\section{Cognitive Deficits Model}

The cognitive deficits model of drug addiction proposes that individuals who develop addictive disorders have abnormalities in an area of the brain called the prefrontal cortex (PFC). The PFC is important for regulation of judgment, planning, and other executive functions. To help us overcome some of our impulses for immediate gratification in favor of more important or ultimately more rewarding long-term goals, the PFC sends inhibitory signals to the VTA DA neurons of the mesolimbic reward system.

The cognitive deficits model proposes that PFC signaling to the mesolimbic reward system is compromised in individuals with addictive disorders, and as a result they have reduced ability to use judgment to restrain their impulses and are predisposed to compulsive drug-taking behaviors. Consistent with this model, stimulant drugs such as methamphetamine appear to damage the specific brain circuit- the frontostriatal loop-that carries inhibitory signals from the PFC to the mesolimbic reward system. In addition, a recent study using magnetic resonance spectroscopy showed that chronic alcohol abusers have abnormally low levels of gamma-amino butyric acid (GABA), the neurochemical that the PFC uses to signal the reward system to release less DA (Behar et al., 1999). As well, the cognitive deficits model of drug addiction could explain the clinical observation that heroin addiction is more severe in individuals with antisocial personality disorder - a condition that is independently associated with PFC deficits (Raine et al., 2000). 
FIGURE 2. The Neurobiological Basis of Dependence and Withdrawal

The locus ceruleus (LC) is an area of the brain that is critically involved in the production of opioid dependence and withdrawal. The diagrams show how opioid drugs affect processes in the LC that control the release of noradrenaline (NA), a brain chemical that stimulates wakefulness, muscle tone, and respiration, among other functions.

A. Normally, natural opiatelike chemicals produced by the body link to mu opioid receptors on the surface of neurons. This linkage activates an enzyme that converts a chemical called adenosine triphosphate (ATP) into another chemical, called cyclic adenosine monophosphate (CAMP), which in turn triggers the release of NA. Prior to initiation of opioid drug abuse, the neuron produces enough NA to maintain normal levels of alertness, muscle tone, respiration, etc.

B. When heroin or another opioid drug links to the mu opioid receptors, it inhibits the enzyme that converts ATP to CAMP. As a result, less CAMP is produced, less NA is released. Alertness, muscle tone, and respiration drop, and the acute opioid effects of sedation, shallow breathing, etc., appear.

C. With repeated heroin exposure, the neuron increases its supply of enzyme and ATP molecules. Using these extra raw materials, the neuron can produce enough cAMP to offset the inhibitory effect of the drug and release roughly normal amounts of NA despite the presence of the drug. At this stage, the individual no longer experiences the same intensity of acute opioid effects as in earlier stages of abuse.

D. When heroin is discontinued after chronic abuse, the drug's inhibitory impact is lost. Operating at normal efficiency but with enhanced supplies of converting enzyme and ATP, the neuron produces abnormally high levels of CAMP, leading to excessive release of NA. The patient experiences the clinical symptoms of withdrawal-jitters, anxiety, muscle cramps, etc. If no further drugs are taken, the neuron will largely revert to its predrug condition (panel A) within days or weeks.

\section{A. Baseline: Normal production of NA}

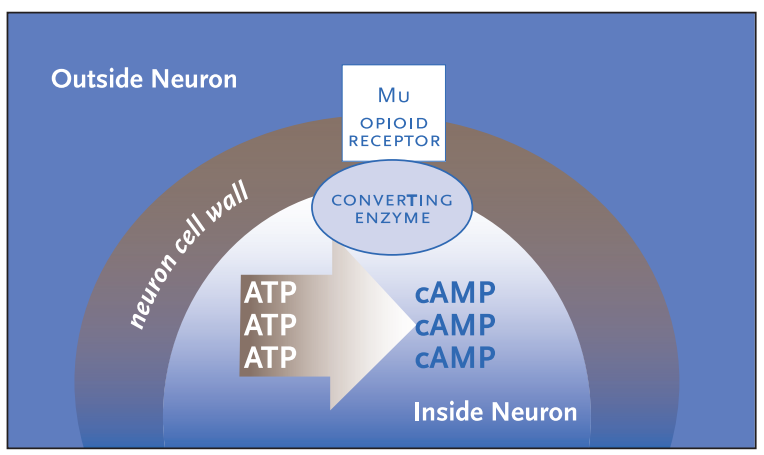

\section{B. Acute opioid inhibition of converting enzyme: Abnormally low production of NA}

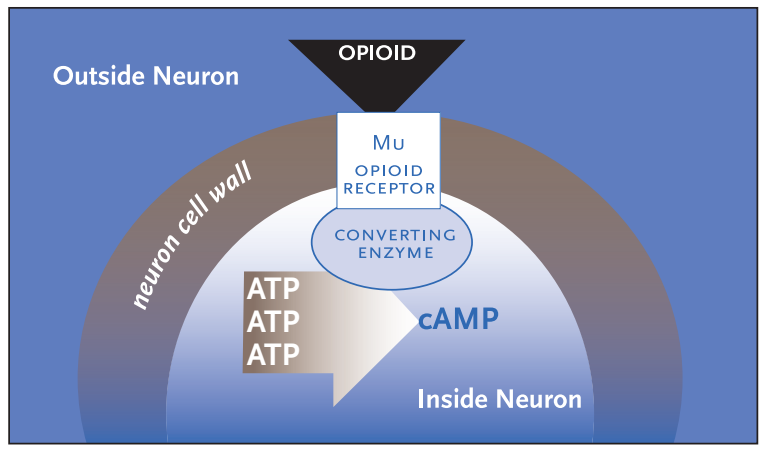

C. Chronic opioid inhibition leads to increased converting enzyme activity: Normal NA level

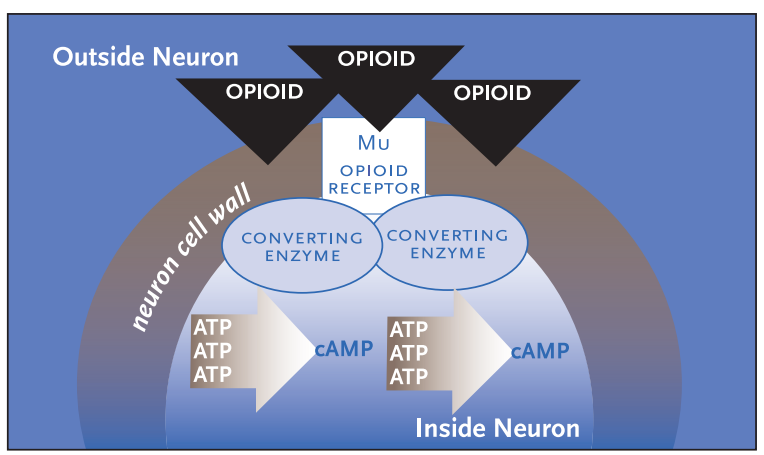

D. Discontinuing opioid leads to increased cyclic AMP due to loss of inhibition: NA excessively high

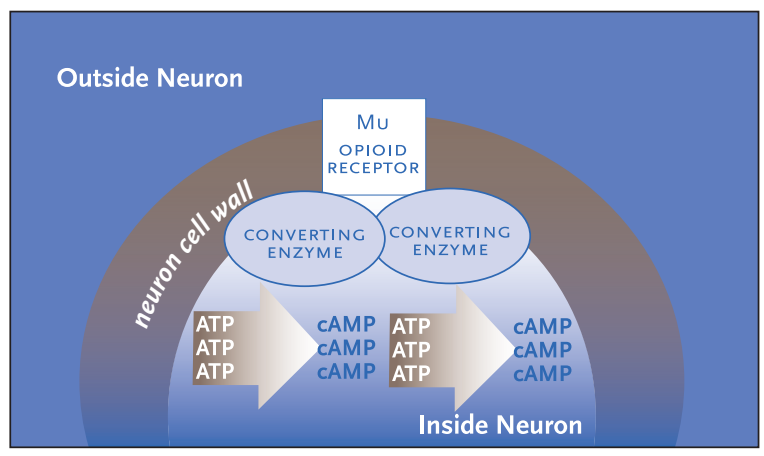

Repeated exposure to opioid drugs induces the brain mechanisms of dependence, which leads to daily drug use to avert the unpleasant symptoms of drug withdrawal. 
Methadone

causes depend-

ence, but-

because of its

steadier influ-

ence on the mu

opioid recep.

tors-it pro-

duces minimal

tolerance and

alleviates crav-

ing and com-

pulsive drug

use.
In contrast to stimulants, heroin apparently damages the PFC but not the fronto-striatal loop. Therefore, individuals who become heroin addicts may have some PFC damage that is independent of their opioid abuse, either inherited genetically or caused by some other factor or event in their lives. This preexisting PFC damage predisposes these individuals to impulsivity and lack of control, and the additional PFC damage from chronic repeated heroin abuse increases the severity of these problems (Kosten, 1998).

\section{STRESS AND DRUG CRAVING}

That drug abuse patients are more vulnerable to stress than the general population is a clinical truism. In the research arena, numerous studies have documented that physical stressors (such as footshock or restraint stress) and psychological stressors can cause animals to reinstate drug use and that stressors can trigger drug craving in addicted humans (e.g., Shaham et al., 2000). The likely explanation for these observations is that opioids raise levels of cortisol, a hormone that plays a primary role in stress responses; and cortisol, in turn, raises the level of activity in the mesolimbic reward system (Kreek and Koob, 1998). By these mechanisms, stress may contribute to the abuser's desire to take drugs in the first place and to his or her subsequent compulsion to keep taking them.

\section{PHARMACOLOGICAL INTERVENTIONS AND TREATMENT IMPLICATIONS}

In summary, the various biological models of drug addiction are complementary and broadly applicable to chemical addictions. Long-term pharmacotherapies for opioid dependence and addiction counteract or reverse the abnormalities underlying those conditions, thereby enhancing programs of psychological rehabilitation. Short-term treatments for relieving withdrawal symptoms and increasing abstinence are beyond the scope of this article; instead, we refer readers elsewhere for detailed neurobiological explanations of the various nonopioid-based abstinence initiation approaches such as clonidine and clonidine-naltrexone for rapid detoxification (see O'Connor and Kosten, 1998, and O'Connor et al., 1997).

The medications most commonly used to treat opioid abuse attach to the brain cells' mu opioid receptors, like the addictive opioids themselves. Methadone and LAAM stimulate the cells much as the illicit opioids do, but they have different effects because of their different durations of action. Naltrexone and buprenorphine stimulate the cells in ways quite distinct from the addictive opioids. Each medication can play a role in comprehensive treatment for opioid addiction.

\section{Methadone}

Methadone is a long-acting opioid medication. Unlike morphine, heroin, oxycodone, and other addictive opioids that remain in the brain and body for only a short time, methadone has effects that last for days. Methadone causes dependence, but-because of its steadier influence on the mu opioid receptors-it produces minimal tolerance and alleviates craving and compulsive drug use. In addition, methadone therapy tends to normalize many aspects of the hormonal disruptions found in addicted individuals (Kling et al., 2000; Kreek, 2000; Schluger et al., 2001). For example, it moderates the exaggerated cortisol stress response (discussed above) that increases the danger of relapse in stressful situations.

Methadone treatment reduces relapse rates, facilitates behavioral therapy, and enables patients to concentrate on life tasks such as maintaining relationships and holding jobs. Pioneering studies by Dole, Nyswander, and Kreek in 1964 to 1966 established methadone's efficacy (Dole et al., 1966). As a Drug Enforcement Administration schedule II controlled substance, the medication is administered primarily in federally regulated methadone programs, where careful monitoring of patients' urine and regular drug counseling are critical components of rehabilitation. Patients are generally started on a daily dose of $20 \mathrm{mg}$ to $30 \mathrm{mg}$, with increases of $5 \mathrm{mg}$ to $10 \mathrm{mg}$ until a dose of $60 \mathrm{mg}$ to $100 \mathrm{mg}$ per day is achieved. The higher doses produce full suppression of opioid craving and, consequently, opioid-free urine tests (Judd et al., 1998). Patients generally stay on methadone for 6 months to 3 years, some much longer. Relapse is common among patients who discontinue methadone after only 2 years or less, and many patients have benefited from lifelong methadone maintenance.

\section{LAAM}

A longer acting derivative of methadone, LAAM can be given three times per week. Recent concerns about heart rhythm problems (specifically, prolonged QT interval) have limited LAAM's use (U.S. Food and Drug Administration, 2001). Nevertheless, longterm maintenance on moderate to high doses of LAAM 
can, like methadone maintenance, normalize physiological functions such as the cortisol stress response (Kling et al., 2000; Kreek, 1992, 2000; Schluger et al., 2001). Dosing with LAAM is highly individualized, and three-times-weekly doses range from $40 \mathrm{mg}$ to $140 \mathrm{mg}$.

\section{Naltrexone}

Naltrexone is used to help patients avoid relapse after they have been detoxified from opioid dependence. Its main therapeutic action is to monopolize mu opioid receptors in the brain so that addictive opioids cannot link up with them and stimulate the brain's reward system. Naltrexone clings to the mu opioid receptors 100 times more strongly than opioids do, but it does not promote the brain processes that produce feelings of pleasure (Kosten and Kleber, 1984). An individual who is adequately dosed with naltrexone does not obtain any pleasure from addictive opioids and is less motivated to use them.

Before naltrexone treatment is started, patients must be fully detoxified from all opioids, including methadone and other treatment medications; otherwise, they will be at risk for severe withdrawal. Naltrexone is given at $50 \mathrm{mg}$ per day or up to $200 \mathrm{mg}$ twice weekly. Patients' liver function should be tested before treatment starts, as heroin abusers may have experienced elevation of certain liver enzymes (transaminases) caused by infectious complications of intravenous drug use, such as hepatitis (Verebey and Mule, 1986).

Unfortunately, medication compliance is a critical problem with naltrexone, because unlike methadone or LAAM, naltrexone does not itself produce pleasurable feelings. Poor compliance limits naltrexone's utility to only about 15 percent of heroin addicts (Kosten and Kleber, 1984).

Naltrexone is also sometimes used to rapidly detoxify patients from opioid dependence. In this situation, while naltrexone keeps the addictive opioid molecules away from the mu opioid receptors, clonidine may help to suppress the excessive NA output that is a primary cause of withdrawal (Kosten, 1990).

\section{Buprenorphine}

Buprenorphine's action on the mu opioid receptors elicits two different therapeutic responses within the brain cells, depending on the dose. At low doses buprenorphine has effects like methadone, but at high doses it behaves like naltrexone, blocking the receptors so strongly that it can precipitate withdrawal in highly dependent patients (that is, those maintained on more than $40 \mathrm{mg}$ methadone daily).

Buprenorphine is expected to be approved by the Food and Drug Administration for the treatment of opioid dependence in 2002. Several clinical trials have shown that when used in a comprehensive treatment program with psychotherapy, buprenorphine is as effective as methadone, except for patients with heroin addiction so severe they would require a dose of more than 100 mg daily (Kosten et al., 1993; Oliveto et al., 1999; Schottenfeld et al., 1997). Buprenorphine offers a safety advantage over methadone and LAAM, since high doses precipitate withdrawal rather than the suppression of consciousness and respiration seen in overdoses of methadone, LAAM, and the addictive opioids. Buprenorphine can be given three times per week. Because of its safety and convenient dosing, it may be useful for treating opioid addiction in primary care settings, which is especially helpful since most opioid addicts have significant medical problems (for example, hepatitis $\mathrm{B}$ or $\mathrm{C}$ and HIV infection). Buprenorphine will be available in $4 \mathrm{mg}$ and $8 \mathrm{mg}$ tablets. A combination tablet with naloxone (Suboxone) has been developed to negate the reward a user would feel if he or she were to illegally divert and inject the medication. The maintenance dose of the combination tablet can be up to $24 \mathrm{mg}$ and used for every-other-day dosing.

As office-based treatment of heroin addiction becomes available, the highest possible safety level (that is, minimal side effects) should be balanced with treatment effectiveness. The patient taking methadone must either visit the medical office daily (not feasible in most cases) or be responsible for taking daily doses at home, as scheduled. Accordingly, for an opioiddependent patient who cannot be relied upon to take the medication as instructed and thus might overdose, buprenorphine in three doses weekly would be a safer choice than methadone. The patient's office visits could be limited to once or twice per week, with remaining buprenorphine doses taken at home. Also, buprenorphine has less overdose potential than methadone, since it blocks other opioids and even itself as the dosage increases.

\section{SUMMARY}

Opioid dependence and addiction are most appropriately understood as chronic medical disorders, like hypertension, schizophrenia, and diabetes. As with those other diseases, a cure for drug addiction is unlikely, 
and frequent recurrences can be expected; but longterm treatment can limit the disease's adverse effects and improve the patient's day-to-day functioning.

The mesolimbic reward system appears to be central to the development of the direct clinical consequences of chronic opioid abuse, including tolerance, dependence, and addiction. Other brain areas and neurochemicals, including cortisol, also are relevant to dependence and relapse. Pharmacological interventions for opioid addiction are highly effective; however, given the complex biological, psychological, and social aspects of the disease, they must be accompanied by appropriate psychosocial treatments. Clinician awareness of the neurobiological basis of opioid dependence, and information-sharing with patients, can pro- vide insight into patient behaviors and problems and clarify the rationale for treatment methods and goals.

\section{ACKNOWLEDGMENT}

This work was supported by NIDA grants number P50-DA-1-2762, K05-DA-0-0454, K12-DA-0-0167, R01-DA-1-3672, and R01-DA-1-4039.

\section{CORRESPONDENCE}

Thomas R. Kosten, M.D.,VA Connecticut Healthcare System, Department of Psychiatry 151D, 950 Campbell Avenue, Bldg. 35, West Haven, CT 06516; e-mail: thomas.kosten@yale.edu.

\section{REFERENCES}

Behar, K.L., et al., 1999. Preliminary evidence of low cortical GABA levels in localized $1 \mathrm{H}-\mathrm{MR}$ spectra of alcohol-dependent and hepatic encephalopathy patients. American Journal of Psychiatry 156(6):952-954.

Breiter, H.C., et al., 1997. Acute effects of cocaine on human brain activity and emotion. Neuron 19(3):591-611.

Dole, V.P.; Nyswander, M.E.; Kreek, M.J., 1966. Narcotic blockade. Archives of Internal Medicine 118 (4):304-309.

Grace, A.A., 2000. The tonic/phasic model of dopamine system regulation and its implications for understanding alcohol and stimulant craving. Addiction 95(Suppl 2):S119-S128. Judd, L.L., et al., 1998. Effective medical treatment of opiate addiction. Journal of the American Medical Association 280(22):1936-1943.

Kling, M.A., et al., 2000. Opioid receptor imaging with PET and [18F] cyclofoxy in long-term, methadone-treated former heroin addicts. Journal of Pharmacological and Experimental Therapeutics 295(3):1070-1076.

Koob, G.F., and LeMoal, M., 2001. Drug addiction, dysregulation of reward, and allostasis. Neuropsychopharmacology 24(2):97-129.

Kosten, T.R., 1990. Neurobiology of abused drugs: Opioids and stimulants. Journal of Nervous and Mental Disease 178(4):217-227.

Kosten, T.R., 1998. Pharmacotherapy of cerebral ischemia in cocaine dependence. Drug and Alcohol Dependence 49(2):133-144.

Kosten, T.R., and Kleber, H.D., 1984. Strategies to improve compliance with narcotic antagonists. American Journal of Drug and Alcohol Abuse $10(2): 249-266$.

Kosten, T.R.; Schottenfeld, R.S.; Ziedonis, D.; and Falcioni, J., 1993. Buprenorphine versus methadone maintenance for opioid dependence. Journal of Nervous and Mental Disease $181(6): 358-364$.

Kreek, M.J., 1992. Rationale for maintenance pharmacotherapy of opiate dependence. In C.P. O'Brien and J.H. Jaffe (eds.), Addictive States. New York: Raven Press, pp. 205-230.

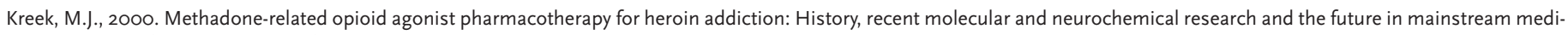
cine. Annals of the NY Academy of Sciences 909:186-216.

Kreek, M.J., and Koob, G.F., 1998. Drug dependence: Stress and dysregulation of brain reward pathways. Drug and Alcohol Dependence 51 (1-2):23-47.

O'Connor, P.G., and Kosten, T.R., 1998. Rapid and ultrarapid opioid detoxification techniques. Journal of the American Medical Association 279(3):229-234.

O'Connor, P.G., et al., 1997. Three methods of opioid detoxification in a primary care setting: A randomized trial. Annals of Internal Medicine 127(7):526-530.

Oliveto, A.H., et al., 1999. Desipramine in opioid-dependent cocaine abusers maintained on buprenorphine versus methadone. Archives of General Psychiatry 56 (9):812-820.

Raine, A., et al., 2000. Reduced prefrontal gray matter volume and reduced autonomic activity in antisocial personality disorder. Archives of Ceneral Psychiatry 57(2):119-127.

Robinson, T.E., and Berridge, K.C., 2000. The psychology and neurobiology of addiction: An incentive-sensitization view. Addiction 95(Suppl 2):S91-S117.

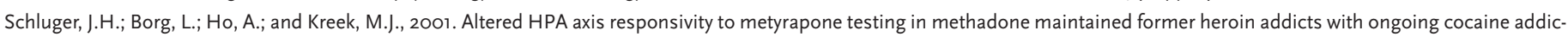
tion. Neuropsychopharmacology 24(5):568-575.

Schottenfeld, R.S., et al., 1997. Buprenorphine versus methadone maintenance treatment for concurrent opioid dependence and cocaine abuse. Archives of General Psychiatry 54(8): $713-720$.

Shaham, Y.; Erb, S.; and Stewart, J., 2000. Stress-induced relapse to heroin and cocaine seeking in rats: A review. Brain Research Reviews 33(1):13-33.

U.S. Food and Drug Administration, 2001. "FDA Talk Paper: FDA Announces Labeling Changes Following Cardiac Adverse Events With Addiction Drug." April 20, 2001. [www.fda.gov/bbs/topics/ANSWERS/2001/ANS01076.html]

Verebey, K.G., and Mule, S.J., 1986. Naltrexone (Trexan): A review of hepatotoxicity issues. In L.S. Harris (ed.), Problems of Drug Dependence, 1985: Proceedings of the 47th Annual Scientific Meeting, the Committee on Problems of Drug Dependence, Inc. NIDA Research Monograph 67. DHHS Publication No. (ADM) 86-1448. Washington, DC: U.S. Government Printing Office, pp. 73-81. 